\section{Deep down}

Tumours evolve as they develop, making them heterogeneous and increasing the probability that subclones resistant to specific types of therapy will be selected for during treatment. If all subclones within a tumour could be identified, one could then identify the important 'driver' mutations that have evolved within the tumour. Peter Campbell, Mike Stratton and colleagues have used ultra-deep pyrosequencing to identify related clones in patients with chronic lymphocytic leukaemia (CLL).

On contact with antigen, B cells undergo somatic hypermutation in order to vary the 'fit' of the B-cell receptor. CLL is a clonal B-cell tumour in which each patient has a unique $\mathrm{V}(\mathrm{D}) \mathrm{J}$ rearrangement of

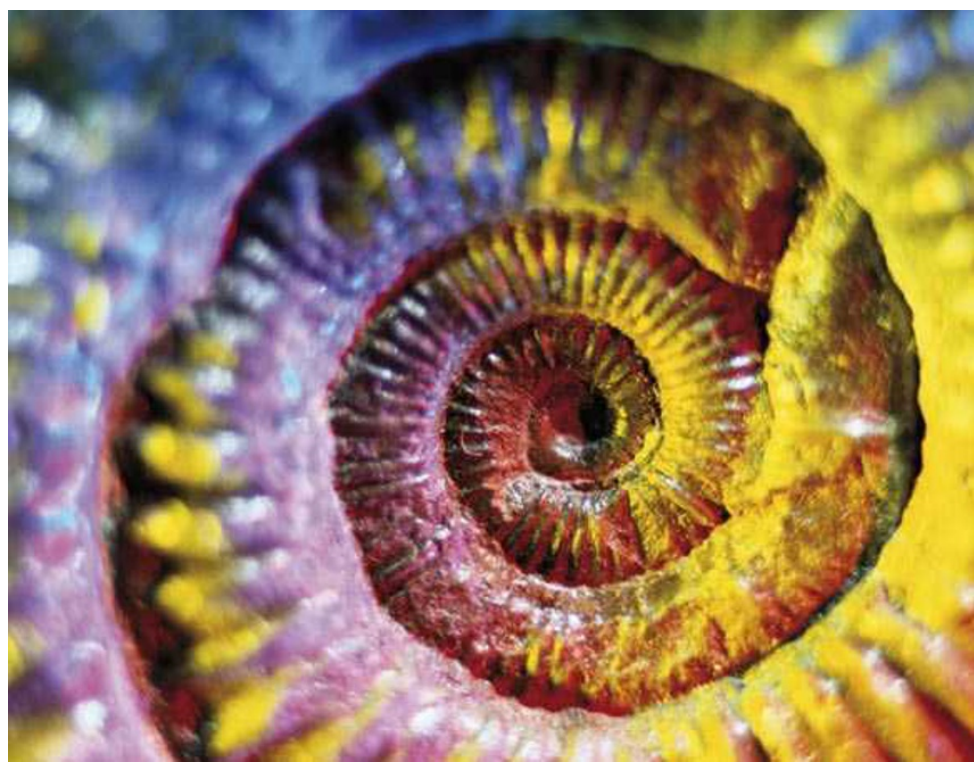

the immunoglobulin heavy chain $(\underline{I G H})$ locus, driven by somatic hypermutation. Some patients show excessive somatic hypermutation in a 300 bp sequence within $I G H$ and evidence indicates that some of these changes can contribute to patient prognosis. Campbell and colleagues used massively parallel sequencing to investigate the mutations at this site in 22 patients with CLL. The authors used nested PCR to amplify the patient-specific variant regions of $I G H$, and these were sequenced using pyrosequencing. As each bead used in this massively parallel sequencing platform represents a single DNA molecule, each in fact denotes an $I G H$ haplotype from a single leukaemic cell. The germline (non-rearranged) $I G H$ locus was also sequenced and used to identify PCR and sequencing errors. Such errors happily conformed to an identifiable pattern, making it possible to eliminate these changes in sequence as artefacts and not owing to genetic evolution. As a result, careful sequence analysis was able to identify rare genetic clones, down to a level of 1 in 5,000 .

Of the samples, $25 \%$ had $>1$ clone, with the number varying from 2 to 18 , and much of the variation seen within the $300 \mathrm{bp}$ region was consistent with changes induced by the somatic hypermutation machinery. For 2 of the patient samples, the authors constructed phylogenetic trees detailing how the clones had evolved within the tumour. There seemed to be 3 different subclasses of clones: intermediate stages en route to the dominant clone; divergent evolution from a common ancestor in the form of blind alleys; and ongoing evolution of the dominant clone.

CLL is particularly amenable to this type of study as the mutations occur in an area of DNA that is short enough to be fully sequenced in a single read. However, the authors hope that this technique can be applied to other tumour types and perhaps be used to understand tumour evolution during invasion and metastasis.

\section{Nicola McCarthy}

ORIGINAL RESEARCH PAPER Campbell, P. J. et al. Subclonal phylogenetic structures in cancer revealed by ultra-deep sequencing. Proc. Natl Acad. Sci. USA 105, 13080-13085 (2008) 